\title{
On the Spectrum of the Space of Bounded Holomorphic Functions on Strongly Pseudoconvex Domains in $C^{n}$
}

Kenzō ADACHI*

Let $D$ be a bounded strongly pseudoconvex domain in $C^{n}$ with $C^{3}$-class boundary. Let $H^{\infty}(D)$ be the space of bounded holomorphic functions on $D$. We denote the spectrum of $H^{\infty}(D)$ by $\Sigma H^{\infty}(D)$. Then the following theorem holds.

THEOREM. $D$ is open in $\sum H^{\infty}(D)$.

REMARK. If $S$ is a unit polydisc in $C^{n}$, then $S$ is open in $\sum H^{\infty}(S)$ (see [1]). If $S$ is a ball or a special analytic polyhedron, then $S$ is open in $\sum H^{\infty}(S)$ (see [4]).

Lemma 1. Let $F$ be the sheaf of germs of bounded holomorphic functions on $D$, Then $H^{p}(\bar{D}, F)=0$ for $p \geqq 1$.

PROOF. This is the result of Lieb [3].

Lemma 2. Let $a \in D$ and $f \in H^{\infty}(D)$. Then there exists $f_{1}, \ldots, f_{n} \in$ $H^{\infty}(D)$ such that $f(z)=\sum_{k=1}^{n} f_{k}(z)\left(z_{k}-a_{k}\right)$ if $f(a)=0$.

Proof. The proof is the same as the proof of the theorem 1 of Kerzman and Nagel [2].

Proof of the Theorem.

For any $\phi \in H^{\infty}(D)$, we define

$$
\pi(\phi)=\phi(z)=\left(\phi\left(z_{1}\right), \ldots, \phi\left(z_{n}\right)\right) .
$$

Received October 16, 1972.

*Department of Mathematics, Faculty of Science, Ibaraki University, Mito. 
Then $\pi$ is continuous. For $\lambda \in D$, we define $\phi_{2}(f)=f(\lambda)$ for all $f \in H^{\infty}$ $(D)$. Then we have $\phi_{2} \in \sum H^{-}(D)$ and $\pi\left(\phi_{2}\right)=\phi_{2}(z)=\lambda$. Suppose that $\pi(\phi)=\lambda \in D$ and $f \in H^{-}(D)$. If $f$ vanishes at $\lambda$, then from Lemma 2, there exists $f_{1}, \ldots, f_{n} \in H^{\infty}(D)$ such that $f(z)=\sum_{k=1}^{n}\left(z_{k}-\lambda_{k}\right) f_{k}(z)$. Then we have

$$
\phi(f)=\sum_{k=1}^{n}\left(\phi\left(z_{k}\right)-\lambda_{k}\right) \phi\left(f_{k}(z)\right)=0 .
$$

This shows that $\pi$ is one to one over $D$. Let $\pi^{-1}(D)=\Delta$, then $\pi$ maps $\Delta$ homeomorphically onto $D$. Therefore our theorem is proved.

\section{References}

[1] K. Hoffman, Banach spaces of analytic functions, Prentice-Hall, INC., Englwood Cliffs, N. J., (1962).

[2] N. Kerzman and A. Nagel, On finitely generated ideals in certain function algebras, J. Funct. Anal., 7 (1971), 212-215.

[3] I. Lieb, Die Cauchy-Riemannschen Differentialgleichungen auf streng pseudokonvexen Gebieten, Math. Ann., 190 (1970), 6-44.

[4] E. L. Stout, Some remarks on varieties in polydiscs and bounded holomorphic functions, Pacific J. Math., 32 (1970), 813-820. 